\title{
Diferenciais da prática contraceptiva no Município de São Paulo, Brasil: resultados do inquérito populacional Ouvindo Mulheres
}

\author{
Differences in contraceptive practice in the \\ city of São Paulo, Brazil: results of the Ouvindo \\ Mulheres population survey
}

\section{Diferenciales de la práctica contraceptiva en el Municipio de São Paulo, Brasil: resultados de la encuesta poblacional Ouvindo Mulheres}

\section{Resumo}

A contracepção é fundamental para que as mulheres possam regular sua fecundidade, exercendo uma das dimensões dos direitos reprodutivos. No entanto, desconhecemos como elas enfrentam esse desafio na maior cidade do Brasil, São Paulo. Para preencher essa lacuna, o inquérito populacional Ouvindo Mulheres: Contracepção no Município de São Paulo foi realizado junto a uma amostra probabilística de 4 mil mulheres com 15 a 44 anos de idade, residentes nessa cidade, em 2015. Neste artigo, apresenta-se a prevalência da prática contraceptiva, analisam-se os fatores associados ao não uso de contracepção e aos tipos de contraceptivos em uso. A prevalência da anticoncepção foi estimada para mulheres com, pelo menos, uma relação heterossexual nos 12 meses anteriores à entrevista e que não estavam grávidas. Regressão logística foi utilizada para verificar fatores associados ao não uso de contracepção, e o modelo CHAID, para identificar associações aos tipos de contraceptivo em uso. A prevalência da anticoncepção foi 84,8\% (IC95\%: 83,2-86,3). Os contraceptivos mais prevalentes foram pílula e preservativo masculino. Associaram- se ao não uso de anticoncepção, religião (Pentecostal), número de filhos (menos do que 3), não ter usado contraceptivo na primeira relação sexual, não ter parceiro e não ter tido relação sexual no mês anterior. O número de filhos tidos e a idade da mulher foram os dois primeiros níveis de discriminação dos tipos de contraceptivo utilizados. A prevalência da anticoncepção é alta, mas mantém-se a concentração em dois métodos: anteriormente, laqueadura e pílula, agora, pílula e preservativo masculino. É necessário incorporar novos contraceptivos hormonais no Sistema Único de Saúde (SUS) e promover o uso de métodos de longa duração como o DIU.

Anticoncepção; Direitos Sexuais e Reprodutivos; Inquéritos Populacionais
Tania Di Giacomo do Lago 1

Suzana Kalckmann 1

Maria Cecília Goi Porto Alves 1

Maria Mercedes Loureiro Escuder 1

Mitti Koyama 2

Regina Maria Barbosa 3

doi: 10.1590/0102-311X00096919

\author{
orrespondência \\ T. D. G. Lago \\ Instituto de Saúde, Secretaria de Estado da Saúde de São Paulo. \\ Rua Santo Antonio 590, São Paulo, SP 01314-000, Brasil. \\ tania_di@uol.com.br \\ 1 Instituto de Saúde, Secretaria de Estado da Saúde de São \\ Paulo, São Paulo, Brasil. \\ ${ }^{2}$ Kamiyama Consultoria Estatística, São Paulo, Brasil. \\ 3 Núcleo de Estudos de População, Universidade Estadual de \\ Campinas, Campinas, Brasil.
}




\section{Introdução}

O primeiro estudo a abordar a anticoncepção feminina na cidade de São Paulo, Brasil, ocorreu em 1965 e identificou que 6 a $8 \%$ das mulheres com 15 a 49 anos em união estavam esterilizadas 1 . Posteriormente, Nakamura 2 identificou que 63,4\% das mulheres paulistanas casadas, com 15 e 44 anos, faziam contracepção em 1978. A prevalência da pílula era 30\%; da laqueadura, 14\%; comportamentais, 10,4\%; e do preservativo masculino, $6,9 \%$.

A atenção à anticoncepção só foi introduzida no sistema público de saúde em 1984, quando diafragma e DIU foram ofertados à maioria da população pela primeira vez ${ }^{3}$. Esperava-se, com isso, ampliar as opções contraceptivas disponíveis para as mulheres brasileiras 4,5 e, consequentemente, reduzir a necessidade de recorrer a um método cirúrgico, na prática, irreversível, que viria provocar arrependimento em muitas mulheres e, ainda, induzir ao parto cirúrgico ${ }^{6}$.

O primeiro inquérito populacional de abrangência nacional, realizado em 1986 , identificou $43 \%$ das mulheres com 15 a 44 anos praticando (ela/ou parceiro) anticoncepção; percentual que alcançava $66 \%$ entre aquelas que viviam com o companheiro. Essa frequência era semelhante à encontrada em países industrializados, mas o "mix de contraceptivos" em uso (como se convencionou internacionalmente denominar a composição da prática segundo tipo de método em uso e sua proporção entre as usuárias) era substancialmente diferente por se concentrar em dois métodos: a esterilização feminina e a pílula, utilizadas, respectivamente, por $27 \%$ e $25 \%$ das mulheres naquela faixa etária e unidas 7.

A Pesquisa Nacional de Demografia e Saúde, realizada em 1996, viria mostrar o aumento da prevalência da anticoncepção no Brasil para 76\% das mulheres com 15 a 44 anos vivendo em união, com maior concentração nos dois contraceptivos referidos, decorrente da elevação da porcentagem de mulheres esterilizadas, que alcançou 38,5\% 8. Naquele ano, foi aprovada, no Congresso Nacional, a lei que regulamenta o planejamento familiar e o acesso à esterilização, no sentido de desencorajar a cirurgia precoce e abrir a possibilidade de sua realização independente do momento do parto 9 .

Novo inquérito populacional, realizado em 2006 10, indicou elevação da frequência da anticoncepção e surpreendeu ao revelar o declínio da prevalência da esterilização feminina para $25,9 \%$ entre mulheres unidas com idade entre 15 e 44 anos e maior diversificação da prática anticoncepcional 11.

A cidade de São Paulo, onde vivem cerca de 2,8 milhões de mulheres em idade fértil 12, carecia dessas informações há 40 anos. Um inquérito populacional intitulado Ouvindo Mulheres: Contracepção no Município de São Paulo foi realizado a fim de identificar as prevalências das práticas contraceptivas e os fatores a elas associados.

\section{Metodologia}

Estudo de corte transversal foi conduzido junto a uma amostra probabilística de 4 mil mulheres com 15 a 44 anos de idade, residentes na cidade de São Paulo, em 2015. As unidades amostrais foram selecionadas em dois estágios. No primeiro, foram sorteados os setores censitários, e, no segundo, domicílios particulares permanentes. Todas as mulheres naquela faixa etária, residentes nos domicílios selecionados, foram convidadas a participar do estudo (elegíveis). A amostra foi estratificada segundo Coordenadoria Regional de Saúde (CRS) do município, tendo sido sorteados 30 setores censitários em cada uma. Responderam ao estudo $75 \%$ dos domicílios ocupados e $77 \%$ das mulheres elegíveis, totalizando 3.895 mulheres, distribuídas em 3.081 domicílios. Os dados foram coletados por meio de entrevistas "face a face", por entrevistadoras (mulheres), após as participantes receberem os esclarecimentos sobre o estudo, concordarem e assinarem o Termo de Consentimento. Para as menores de 18 anos, obteve-se o Termo de Assentimento. As informações foram registradas em tabletes com aplicativo desenvolvido para o estudo e transferidas para o banco de dados via Internet. Informações mais detalhadas da metodologia foram descritas anteriormente 13 .

A prevalência da anticoncepção foi estimada para mulheres que tiveram, pelo menos, uma relação heterossexual nos 12 meses anteriores à entrevista e que não estavam grávidas nesse momento. Ela foi medida pela porcentagem de mulheres que responderam afirmativamente à pergunta "Você e/ ou seu parceiro fazem alguma coisa ou usam algum método para evitar gravidez?”. O contraceptivo em uso foi o método referido pela mulher. Quando mais de um contraceptivo foi mencionado, o de 
maior eficácia foi selecionado, conforme critério utilizado pelas pesquisas de demografia e saúde 14, exceto no caso da associação de pílula e preservativo masculino, que foi considerada como um "tipo de contracepção", em virtude da alta frequência encontrada.

Para identificar os principais fatores associados ao não uso de contracepção, foram excluídas da análise as mulheres que disseram não estar praticando anticoncepção por querer engravidar ou pela existência de diagnóstico médico de infertilidade dela ou do parceiro. As variáveis consideradas foram: (a) características sociodemográficas - CRS de residência, idade, cor autodeclarada, grau completo de escolaridade, religião atual, possuir renda própria, ter plano de saúde e classe familiar de consumo segundo o Critério Brasil de Classificação Econômica de 2013 (Associação Brasileira de Empresas de Pesquisa. httpp://www.abep.org/Servicos/Download.aspx?id=0, acessado em 14/Out/2016); (b) parceria e vida sexual - idade à primeira relação sexual, ocorrência de atividade sexual nos últimos 30 dias, número de parceiros sexuais na vida, existência de parceiro atual; (c) experiência reprodutiva - idade na primeira gestação, número de filhos nascidos vivos; (d) acesso a serviços de saúde - ter realizado atendimento ginecológico no último ano. As variáveis cuja associação foi significante ao nível de 5\% na análise univariada foram submetidas à regressão logística múltipla. Inicialmente, todas essas foram incluídas no modelo, e, em seguida, as não significantes ao nível de $5 \%$ foram excluídas uma a uma por ordem de significância (método backward stepwise).

Para identificar diferentes perfis de usuárias segundo a composição do mix contraceptivo e a combinação de fatores que os caracterizam, foi empregada a árvore de decisão utilizando o algoritmo CHAID (chi-squared automatic interaction detector) 15 que permite identificar grupos homogêneos de indivíduos por comparação sistemática de suas características. Seu objetivo é estabelecer relação entre estas características (variáveis explicativas) e uma única variável resposta (variável dependente). A árvore de decisão é ajustada por meio de sucessivas divisões no conjunto de dados com o intuito de tornar os subconjuntos cada vez mais homogêneos em relação à variável resposta. O processo de divisão é repetido até nenhuma das variáveis selecionadas mostrarem influência significativa na divisão ou quando o tamanho do subconjunto for muito pequeno. Neste estudo, a variável dependente foi o tipo de contraceptivo em uso, e as variáveis independentes foram as mesmas anteriormente descritas nos itens (a), (b), (c) e (d). Mulheres que não estavam em uso de contracepção foram excluídas desta análise.

Os dados foram ponderados pelo peso do delineamento, visando compensar as probabilidades distintas de seleção utilizadas nas coordenadorias. Esses pesos iniciais sofreram ajustes pelas taxas de resposta, uma vez que a ausência de resposta também não foi uniforme. Esteve associada ao nível socioeconômico do setor censitário, indicado pela renda média dos moradores no Censo Demográfico de 2010 (Instituto Brasileiro de Geografia e Estatística. http://www.ibge.gov.br). Outro ajuste foi introduzido por pós-estratificação, visando igualar a distribuição por faixa etária da amostra àquela observada na população, considerando estimativas da Fundação Sistema Estadual de Análise de Dados (Fundação Seade. https://www.seade.gov.br/) para 2015. As análises estatísticas foram feitas considerando o planejamento amostral complexo: sorteio de conglomerado (setores censitários), estratificação e ponderação (pesos de delineamento, de não resposta e de pós-estratificação por idade). Como não existe rotina implementada para o procedimento CHAID na análise de amostras complexas no software SPSS versão 20.0 (https://www.ibm.com/), a análise foi realizada ponderada utilizando-se o peso de expansão padronizado - a expansão da amostra leva ao tamanho efetivo da amostra.

\section{Resultados}

Das 3.895 entrevistadas, 1.010 mulheres foram excluídas da análise da contracepção atual: $853(21,3 \%)$ por não terem tido, pelo menos, uma relação heterossexual nos últimos 12 meses, $135(3,4 \%)$ por estarem grávidas no momento da entrevista e $22(0,4 \%)$ por respostas inconsistentes que colocavam em dúvida a prática contraceptiva registrada. Dentre as 2.885 incluídas nesta análise, $2.441(84,8 \%)$ disseram estar, elas ou o companheiro, praticando contracepção.

A prática mais frequente foi o uso da pílula (27\%), seguida pelo preservativo masculino (19\%) e pelo hormonal injetável (10,4\%). A associação de pílula e preservativo masculino foi relatada por $8,9 \%$ das mulheres, a laqueadura por $7,5 \%$, pouco superior a da vasectomia (6,2\%). O uso de métodos com- 
portamentais (tabelinha e coito interrompido) e de DIU foi infrequente (2,8 e 2,5\% respectivamente), superando apenas o conjunto de contraceptivos mais recentemente disponíveis no Brasil, representado por 17 mulheres (0,5\%). Incluem-se aí oito usuárias de adesivos, quatro de anel vaginal, três de implantes, uma de camisinha feminina e uma que recorre à pílula do dia seguinte (Tabela 1).

Dentre as 444 mulheres que não estavam praticando anticoncepção, obteve-se o motivo em 434 entrevistas. Dessas, 159 (36,6\%) relataram ocorrência esporádica ou ausência de relações sexuais, 137 (31,8\%) queriam engravidar, 27 (5,9\%) achavam difícil praticar anticoncepção ou temiam efeitos colaterais, 22 (4,9\%) referiram diagnóstico médico de infertilidade conjugal, 19 (4\%) achavam difícil engravidar, e $16(3,4 \%)$ desconheciam como fazer ou onde obter anticoncepção. Outras 44 mulheres apontaram razões diferentes (10,7\%), e dez disseram não saber explicar o motivo $(2,5 \%)$.

$\mathrm{Na}$ análise bivariada, não se verificou associação do uso de contracepção a coordenadoria de saúde de residência da mulher, cor, escolaridade, idade à primeira relação sexual, número de parceiros sexuais na vida ou idade na primeira gestação. O não uso de contracepção associou-se ao grupo etário mais jovem, às religiões pentecostais, à classe de consumo $\mathrm{D} / \mathrm{E}$, à ausência de renda própria da mulher, a não ter plano de saúde, a não ter realizado um atendimento ginecológico no ano anterior, ao fato de não ter usado contraceptivo na primeira relação sexual, a não ter filhos ou ter menos do que 3, a não ter parceiro e ao fato de não ter tido relação sexual nos 30 dias anteriores. Para facilitar a interpretação da interação entre essas duas últimas variáveis, uma terceira foi construída, articulando a existência ou não de parceiro atual e de relações sexuais no mês anterior para o modelo de análise multivariada. Dessa forma, foi possível perceber que cada uma contribui, de forma independente, como se verá a seguir.

À análise multivariada, permaneceram associadas ao não uso de contracepção, a religião (pentecostais versus sem religião: $\mathrm{OR}=0,48$; IC95\%: 0,32-0,72), o não uso de anticoncepcional na primeira relação sexual (OR = 0,61; IC95\%: 0,47-0,79), o número de filhos tidos (nenhum: OR = 0,53; IC95\%: 0,33-0,83 e 1-2: OR = 0,55; IC95\%: 0,36-0,84), não ter realizado consulta ginecológica no ano anterior $(\mathrm{OR}=0,73$; IC95\%: 0,54-0,98) e a nova variável. A chance de as mulheres sem parceiro e que não tiveram relações sexuais no mês anterior estarem em uso de contracepção foi $94 \%$ menor do que para aquelas com parceiro e que tiveram atividade sexual nesse período (OR = 0,06; IC95\%: 0,04-0,09). Tal chance foi $74 \%$ menor para aquelas que tinham parceiro, mas não tiveram relação sexual no mês anterior (OR = 0,26; IC95\%: 0,14-0,46) e para as que tiveram atividade sexual nesse período, mas não tinham um parceiro $(\mathrm{OR}=0,26$; IC95\%: 0,16-0,42) (Tabela 2).

\section{Tabela 1}

Prevalência da prática contraceptiva de mulheres com 15 a 44 anos de idade sexualmente ativas * e não grávidas. Município de São Paulo, Brasil, 2015.

\begin{tabular}{lccc}
\hline Prática contraceptiva & $\mathbf{n}$ & \% & IC95\% \\
\hline Nenhuma & 444 & 15,2 & $13,7-16,8$ \\
Pílula & 760 & 27,0 & $25,2-28,9$ \\
Preservativo masculino & 541 & 19,0 & $17,5-20,6$ \\
Injetáveis & 310 & 10,4 & $9,1-11,8$ \\
Pílula e preservativo masculino & 250 & 8,9 & $7,8-10,1$ \\
Laqueadura & 231 & 7,5 & $6,5-8,7$ \\
Vasectomia & 178 & 6,2 & $5,4-7,2$ \\
Tabelinha e/ou coito interrompido & 81 & 2,8 & $2,2-3,5$ \\
DIU & 73 & 2,5 & $1,8-3,3$ \\
Outras & 17 & 0,6 & $0,4-1,0$ \\
Total & 2.885 & 100,0 &
\end{tabular}

IC95\%: intervalo de 95\% de confiança.

* Pelo menos uma relação heterossexual nos últimos 12 meses. 
Tabela 2

Fatores associados ao uso de métodos contraceptivos entre mulheres sexualmente ativas * não grávidas com 15 a 44 anos. Município de São Paulo, Brasil, 2015.

\begin{tabular}{|c|c|c|c|c|}
\hline Fatores & $\begin{array}{l}\text { OR bruto } \\
\text { (IC95\%) }\end{array}$ & Valor de p & $\begin{array}{l}\text { OR ajustado ** } \\
\qquad(\text { IC95\%) }\end{array}$ & Valor de p \\
\hline Faixa etária (Ref.: 30 a 44 anos) & & $<0,001$ & & - \\
\hline 15 a 19 & $0,52(0,34-0,79)$ & 0,002 & - & - \\
\hline 20 a 29 & $0,92(0,70-1,20)$ & 0,539 & - & - \\
\hline Classe D/E (Ref.: A, B ou C) & $0,51(0,35-0,76)$ & 0,001 & - & - \\
\hline Não tem renda própria & $0,76(0,59-0,98)$ & 0,033 & - & - \\
\hline Religião (Ref.: sem religião) & & $<0,001$ & & 0,001 \\
\hline Católica & $0,98(0,63-1,51)$ & 0,913 & $0,85(0,54-1,35)$ & 0,496 \\
\hline Pentecostal & $0,57(0,39-0,83)$ & 0,004 & $0,48(0,32-0,72)$ & 0,001 \\
\hline Outras & $0,79(0,53-1,18)$ & 0,253 & $0,85(0,53-1,35)$ & 0,480 \\
\hline Número de filhos (Ref.: 3 ou mais) & & $<0,001$ & & 0,012 \\
\hline Nenhum & $0,55(0,39-0,76)$ & $<0,001$ & $0,53(0,33-0,83)$ & 0,006 \\
\hline $1-2$ & $0,70(0,49-1,00)$ & 0,051 & $0,55(0,36-0,84)$ & 0,006 \\
\hline Não usou método na 1a relação & $0,71(0,57-0,89)$ & 0,003 & $0,61(0,47-0,79)$ & $<0,001$ \\
\hline Sem plano de saúde & $0,69(0,53-0,92)$ & 0,011 & - & - \\
\hline Não teve atendimento ginecológico no último ano & $0,62(0,48-0,80)$ & $<0,001$ & $0,73(0,54-0,98)$ & 0,034 \\
\hline Não tem parceiro & $0,12(0,09-0,16)$ & $<0,001$ & & \\
\hline Não teve relação heterossexual nos últimos 30 dias & $0,12(0,09-0,16)$ & $<0,001$ & & \\
\hline $\begin{array}{l}\text { Parceiro e relações heterossexuais nos últimos } 30 \text { dias (Ref.: com } \\
\text { parceiro e com relações heterossexuais nos últimos } 30 \text { dias) }\end{array}$ & & $<0,001$ & & $<0,001$ \\
\hline Sem parceiro e sem relação sexual nos últimos 30 dias & $0,07(0,05-0,09)$ & $<0,001$ & $0,06(0,04-0,09)$ & $<0,001$ \\
\hline Sem parceiro e com relação sexual nos últimos 30 dias & $0,27(0,16-0,46)$ & $<0,001$ & $0,26(0,14-0,46)$ & $<0,001$ \\
\hline Com parceiro e sem relação sexual nos últimos 30 dias & $0,24(0,14-0,39)$ & $<0,001$ & $0,26(0,16-0,42)$ & $<0,001$ \\
\hline
\end{tabular}

IC95\%: intervalo de 95\% de confiança; OR: odds ratio; Ref.: referência.

* Mulheres que tiveram, pelo menos, uma relação heterossexual nos últimos 12 meses. Foram excluídas as que informaram não fazer contracepção pois queriam engravidar e as que referiram diagnóstico médico de infertilidade (ela e/ou ele);

** OR ajustado por todas as demais variáveis presentes no modelo: faixa etária, número de filhos, classe de consumo (Critério Brasil), renda própria, religião, uso de contracepção na primeira relação sexual, ter plano de saúde, consulta ginecológica no último ano, ter parceiro, ocorrência de relações sexuais no último ano.

Aplicando-se a técnica de CHAID para avaliar a associação de fatores que caracterizam os métodos em uso, obteve-se uma árvore com 3 níveis e 18 nós. Para facilitar a visualização, a árvore foi apresentada em três figuras de acordo com o primeiro nível (número de filhos).

O número de nascidos vivos constituiu-se no primeiro nível de discriminação. Entre as mulheres que não tinham filhos nascidos vivos, verificou-se a maior proporção de uso da pílula (39,4\%), do preservativo masculino $(27,4 \%)$ e da associação de ambos $(17,8 \%)$. Laqueadura $(0,45 \%)$ e vasectomia $(1,3 \%)$ foram raros (Figura 1). Entre mulheres com 1 ou 2 filhos, embora sigam predominando a pílula (32,6\%) e o preservativo masculino $(21,8 \%)$, sua participação na prática contraceptiva $(62,5 \%)$ foi menor do que a observada entre mulheres sem filhos (84,6\%). A proporção de usuárias de injetáveis foi maior (14,5\%), tornando-os o terceiro método mais frequente e elevou-se a participação da laqueadura (6,0\%) e da vasectomia 8,6\% (Figura 2). Entre as mulheres com 3 ou mais filhos, a laqueadura foi a prática contraceptiva mais frequente (36,9\%), seguida pela pílula (15\%) e pela vasectomia (14\%) (Figura 3).

No nível seguinte de discriminação, a idade delimitou sete subconjuntos, descritos a seguir, conforme o número de filhos nascidos vivos.

Mulheres sem filhos (Figura 1). Entre as mais novas (15 a 19 anos), predominou o uso de preservativo masculino $(36,6 \%)$ seguido pela pílula $(28,3 \%)$ e pelo uso concomitantemente de pílula e preservativo masculino $(21,4 \%)$. No subconjunto de mulheres de 20 a 29 anos, a pílula respondeu por 
Figura 1

Fatores associados aos tipos de contraceptivos em uso entre usuárias sem filhos nascidos vivos. Modelo CHAID (chi-squared automatic interaction detector). Município de São Paulo, Brasil, 2015.

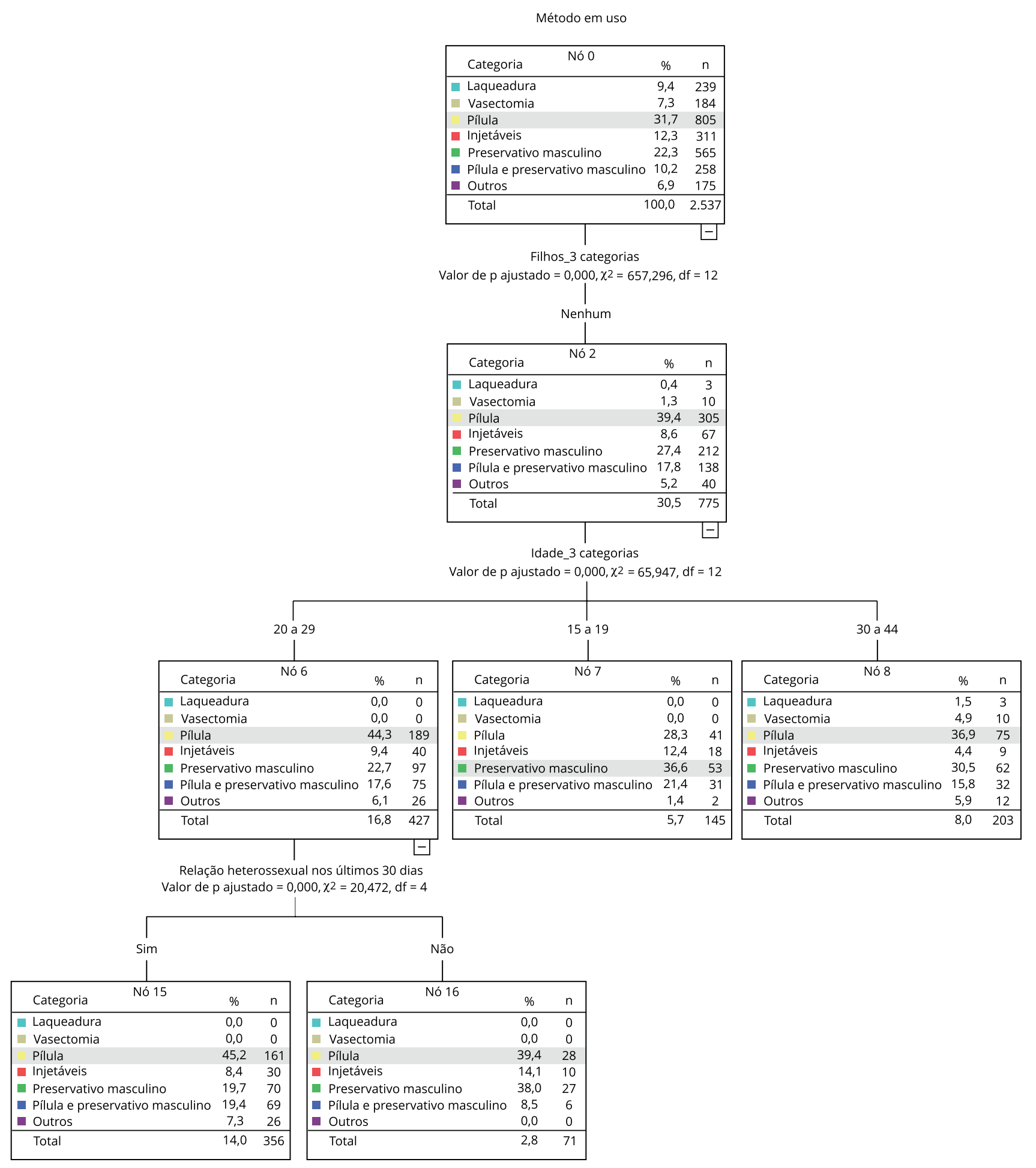




\section{Figura 2}

Fatores associados aos tipos de contraceptivos em uso entre usuárias com 1 a 2 filhos nascidos vivos. Modelo CHAID (chi-squared automatic interaction detector). Município de São Paulo, Brasil, 2015.

Método em uso

\begin{tabular}{|lccc|}
\hline Categoria & Nó 0 & $\%$ & $\mathrm{n}$ \\
\hline Laqueadura & 9,4 & 239 \\
Vasectomia & 7,3 & 184 \\
Pílula & 31,7 & 805 \\
Injetáveis & 12,3 & 311 \\
Preservativo masculino & 22,3 & 565 \\
Pílula e preservativo masculino & 10,2 & 258 \\
Outros & 6,9 & 175 \\
\hline Total & & 100,0 & 2.537 \\
\multicolumn{1}{|l}{} \\
\end{tabular}

Filhos_3 categorias

Valor de $p$ ajustado $=0,000, \chi^{2}=657,296, d f=12$

\begin{tabular}{|c|c|c|}
\hline Categoria & $\%$ & $\mathrm{n}$ \\
\hline Laqueadura & 6,0 & 81 \\
\hline Vasectomia & 8,6 & 115 \\
\hline Pílula & 32,6 & 437 \\
\hline Injetáveis & 14,5 & 195 \\
\hline Preservativo masculino & 21,8 & 292 \\
\hline - Pílula e preservativo masculino & 8,1 & 109 \\
\hline Outros & 8,4 & 113 \\
\hline Total & 52,9 & 1.342 \\
\hline
\end{tabular}

Idade_3 categorias

Valor de $p$ ajustado $=0,000, \chi^{2}=112,471, d f=6$

\begin{tabular}{|c|c|c|c|c|}
\hline \multicolumn{2}{|l|}{20 a $29 ; 15$ a 19} & \multicolumn{3}{|l|}{30 a 44} \\
\hline Categoria & $\%$ & Categoria & $\%$ & $\mathrm{n}$ \\
\hline Laqueadura & 1,6 & Laqueadura & 8,2 & 74 \\
\hline Vasectomia & $1,6 \quad 7$ & Vasectomia & 11,9 & 108 \\
\hline Pílula & $35,4 \quad 154$ & Pílula & 31,2 & 283 \\
\hline Injetáveis & 108 & Injetáveis & 9,6 & 87 \\
\hline Preservativo masculino 2 & 20,9 & Preservativo masculino & 22,2 & 201 \\
\hline Pílula e preservativo masculino & 9,7 & Pílula e preservativo masculino & 7,4 & 67 \\
\hline Outros & $6,0 \quad 26$ & Outros & 9,6 & 87 \\
\hline Total & $17,1 \quad 435$ & Total & 35,8 & 907 \\
\hline
\end{tabular}

Tem plano de saúde Valor de $p$ ajustado $=0,009, \chi^{2}=17,179, \mathrm{df}=6$

\begin{tabular}{|c|c|c|c|c|c|}
\hline \multicolumn{6}{|c|}{ Valor de $p$ ajustado $=0,009, \chi^{2}=17,179, \mathrm{df}=6$} \\
\hline \multicolumn{3}{|l|}{ Sim } & \multicolumn{3}{|l|}{ Não } \\
\hline Categoria & $\%$ & $\mathrm{n}$ & Categoria & $\%$ & $\mathrm{n}$ \\
\hline Laqueadura & 2,4 & 3 & Laqueadura & 1,3 & 4 \\
\hline Vasectomia & 1,6 & 3 & Vasectomia & 1,6 & 5 \\
\hline Pílula & 33,3 & 42 & Pílula & 36,2 & 112 \\
\hline Injetáveis & 17,5 & 22 & E Injetáveis & 27,8 & 86 \\
\hline Preservativo masculino & 19,8 & 25 & Preservativo masculino & 21,4 & 66 \\
\hline Pílula e preservativo masculino & 17,5 & 22 & Pílula e preservativo masculino & 6,5 & 20 \\
\hline Outros & 7,9 & 10 & Outros & 5,2 & 16 \\
\hline Total & 5,0 & 126 & Total & 12,2 & 309 \\
\hline
\end{tabular}

Usou método na 1 ạ relação

Valor de $p$ ajustado $=0,000, \chi^{2}=31,915, d f=6$
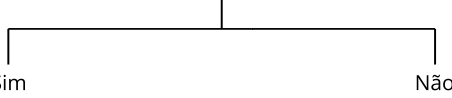

1

\begin{tabular}{|c|c|c|}
\hline Categoria & $\%$ & $n$ \\
\hline Laqueadura & 5,1 & 30 \\
\hline Vasectomia & 11,4 & 67 \\
\hline Pílula & 29,6 & 174 \\
\hline Injetáveis & 10,7 & 63 \\
\hline Preservativo masculino & 25,2 & 148 \\
\hline Pílula e preservativo masculin & 8,3 & 49 \\
\hline Outros & 9,5 & 56 \\
\hline Total & 23,1 & 587 \\
\hline
\end{tabular}

\begin{tabular}{|lcc|}
\hline Categoria & Nó 14 & $\mathrm{n}$ \\
\hline Laqueadura & 13,8 & 44 \\
Vasectomia & 12,8 & 41 \\
Pílula & 34,1 & 109 \\
Injetáveis & 7,5 & 24 \\
Preservativo masculino & 16,6 & 53 \\
Pílula e preservativo masculino & 5,6 & 18 \\
Outros & 9,7 & 31 \\
\hline Total & 12,6 & 320 \\
\hline
\end{tabular}


Figura 3

Fatores associados aos tipos de contraceptivos em uso entre mulheres com 3 ou mais filhos. Modelo CHAID (chi-squared automatic interaction detector). Município de São Paulo, Brasil, 2015.

Método em uso

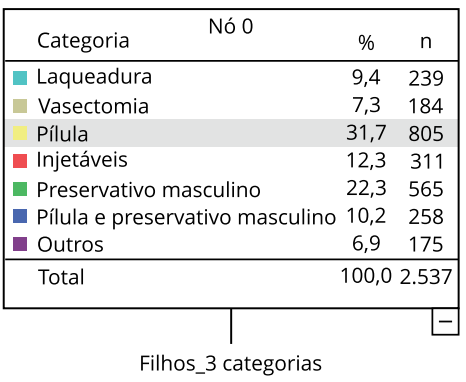

Valor de $p$ ajustado $=0,000, \chi^{2}=657,296, \mathrm{df}=12$

3 ou mais

|

\begin{tabular}{|c|c|c|c|}
\hline & Categoria & $\%$ & $\mathrm{n}$ \\
\hline$\square$ & Laqueadura & 36,9 & 155 \\
\hline 口 & Vasectomia & 14,0 & 59 \\
\hline G & Pílula & 15,0 & 63 \\
\hline $\mathbf{\square}$ & Injetáveis & 11,7 & 49 \\
\hline - & Preservativo masculino & 14,5 & 61 \\
\hline - & Pílula e preservativo masculino & 2,6 & 11 \\
\hline 口 & Outros & 5,2 & 22 \\
\hline & Total & 16,6 & 420 \\
\hline
\end{tabular}

Valor de $p$ ajustado $=0,000, \chi^{2}=55,848, d f=6$

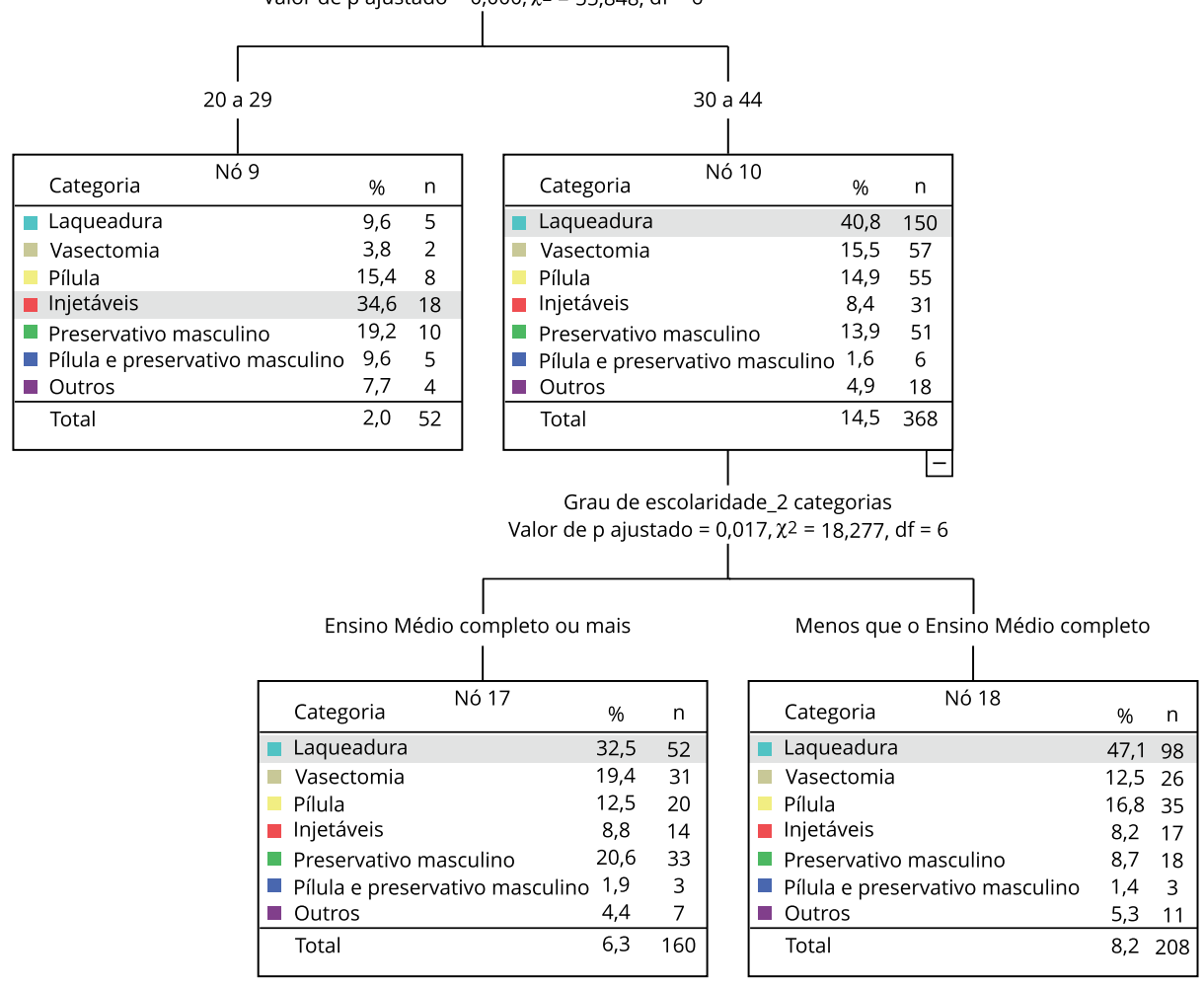


$44,3 \%$ da prática contraceptiva, seguida pelo preservativo masculino $(22,7 \%)$ e pela combinação desses dois métodos (17,6\%), embora em menor participação do que entre as mais jovens. Nessas duas faixas etárias, não foram encontradas usuárias de laqueadura ou com parceiros vasectomizados. Já entre as mais velhas (30 anos ou mais), observou-se a presença de laqueadura $(1,5 \%)$ e de vasectomia $(4,9 \%)$, mantendo-se a pílula como prática mais frequente $(36,9 \%)$, seguida pelo uso do preservativo masculino, seja isolado $(30,5 \%)$ ou associado à pílula $(15,8 \%)$. O fato de ter tido ou não relação sexual nos últimos 30 dias só discriminou o mix contraceptivo para as mulheres na faixa etária de 20 a 29 anos. Entre as que tiveram, houve predomínio da pílula, em uso isolado ( $45,2 \%$ versus $39,4 \%)$ ou concomitante com o preservativo masculino (19,4\% versus $8,5 \%)$. Entre as demais, a participação exclusiva do preservativo masculino foi maior (38\% versus $19,7 \%)$ e muito próxima à da pílula $(39,4 \%)$.

Mulheres com 1 ou 2 filhos (Figura 2). A idade discriminou o grupo em dois subconjuntos: mulheres de 15 a 29 anos e de 30 a 44 anos. A participação da pílula e do preservativo masculino é parecida em ambos. As diferenças que parecem se destacar são a maior proporção de usuárias de injetáveis entre as mulheres com menos de 30 anos $(24,8 \%$ versus $9,6 \%)$ e, entre as mais velhas, a maior presença da laqueadura $(8,2 \%$ versus $1,6 \%)$ e da vasectomia $(11,9 \%$ versus $1,6 \%)$. No terceiro nível de discriminação, variáveis diferentes segmentaram os grupos de mulheres com menos e com mais de 30 anos de idade. A posse de plano de saúde separou as mulheres com menos de 30 anos em dois grupos: dentre as com plano, a participação dos injetáveis diminuiu $(17,5 \%)$ e, entre as sem plano de saúde, os injetáveis fortaleceram-se na segunda posição, $\operatorname{com}(27,8 \%)$ (Figura 2). Entre as mais velhas, ter utilizado ou não contraceptivo na primeira relação sexual determinou dois outros conjuntos de usuárias de anticoncepção. Entre as que disseram não ter se protegido da gravidez, a proporção de esterilizadas foi quase três vezes maior do que entre as que disseram ter se protegido $(13,8 \%$ versus $5,1 \%$ ) (Figura 2 ).

Mulheres com 3 ou mais filhos (Figura 3). Aqui, a idade discriminou apenas dois subconjuntos, por não haver na amostra alguém com menos de 20 anos e 3 filhos. No grupo de mulheres com idade entre 20 a 29 anos, os injetáveis foram o método mais frequentemente utilizado (34,6\% versus $8,4 \%$ entre as usuárias mais velhas). Entre as com 30 a 44 anos, o predomínio da laqueadura intensificou-se (40,8\% versus $9,6 \%$ entre as mais jovens) seguido pela vasectomia (15,5\% versus 3,8 \%). Entre as mais velhas, a influência da escolaridade se fez presente. Para as que haviam completado, pelo menos, o Ensino Médio, observou-se maior participação masculina no mix contraceptivo: 19,4\% das usuárias de anticoncepção tinham parceiros vasectomizados, e 22,5\% referiam uso do preservativo masculino (incluindo uso associado à pílula). Essas proporções foram, respectivamente, 12,5\% e 10,1\% no grupo de menor escolaridade (até médio incompleto).

É importante lembrar que o modelo CHAID não foi utilizado para classificações, mas para explorar possíveis associações entre características das mulheres e diferentes tipos de mix contraceptivos. Se o modelo tivesse sido usado com aquela intenção, a porcentagem de classificação correta teria sido de 36,6\% em função do pequeno número de mulheres utilizando alguns métodos.

\section{Discussão}

A frequência da prática da contracepção entre mulheres de 15 a 44 anos sexualmente ativas foi semelhante à observada no Brasil em 2006 (85,3\%) (Ministério da Saúde. http://bvsms.saude.gov.br/bvs/ pnds/banco_dados.php, acessado em 19/Abr/2016). O principal motivo alegado para não estar em uso de anticoncepção foi a infrequência de relações sexuais, em consonância com achados internacionais ${ }^{16}$. De fato, à análise multivariada, a chance de uma mulher sem parceiro e sem relações sexuais no mês anterior não estar usando contraceptivo foi quase duas vezes a das que tinham parceiro e relações sexuais.

Dentre os fatores associados ao não uso de contracepção, dois fatos chamaram a atenção. A influência da religião na adoção ou não de contracepção não havia sido verificada anteriormente no Brasil. Berquó et al. 17, comparando o uso do preservativo masculino em inquéritos nacionais realizados em 1998 e 2005, identificaram, apenas neste último ano, a influência da religião. No subgrupo de pessoas com relações estáveis, pentecostais e protestantes apresentavam a menor frequência de uso do preservativo masculino. Não se avaliou, no entanto, se esse comportamento se aplicava à pratica contraceptiva ou à necessidade de prevenção de doenças de transmissão sexual, o que é mais provável, 
uma vez que esta última requereria a admissão de outras parcerias sexuais, ainda que anteriores, na vida de um casal. É possível que a "transição religiosa" 18,19 em curso no país, com aumento dos que se declaram evangélicos pentecostais, dos sem religião e com a diminuição do número de católicos, esteja contribuindo para a percepção de comportamentos anteriormente não observados. O crescimento acelerado das chamadas religiões evangélicas pentecostais nos meios de comunicação, mídia, na política tem se associado a posições restritivas do direito reprodutivo 20. Estudos que explorem especificamente a relação entre religiões e contracepção precisam ser realizados no Brasil.

Por outro lado, a associação positiva da situação econômica e da escolaridade da mulher com o uso de anticoncepção verificadas em 1996, e em menor intensidade em 2006 11, não se repetiu em São Paulo. É preciso cautela ao interpretar esse resultado, pois, com o aumento da escolaridade feminina, desigualdades no campo da reprodução têm sido melhor evidenciadas quando se comparam mulheres com menos de 4 anos de estudo e aquelas com 11 ou mais anos 11,21. Isso não foi possível no presente estudo pelo número pequeno de mulheres na categoria inferior de escolaridade.

Comparando-se os tipos de contraceptivos em uso no estudo nacional anterior, observou-se, no munícipio, frequência bem superior de uso de injetáveis (10,4\% versus 4,9\% em 2006), de preservativos masculinos, em uso isolado ou associado à pílula, (28\% versus $20 \%$ em 2006) e, em menor grau, da pílula (36\% versus $30,3 \%$ em 2006$)$.

A diferença mais importante foi a baixa proporção de mulheres laqueadas (7,5\%), quando comparada à encontrada em 2006 no Brasil (23,2\%) e no Sudeste (17,5\%) entre mulheres sexualmente ativas no mesmo grupo etário. Em contrapartida, a esterilização masculina se manteve em nível similar $(6,2 \%)$ à do Sudeste $(6,4 \%)$ naquele ano. A possibilidade de uma demanda frustrada pela laqueadura tubária já foi apontada e relacionada ao pequeno número de serviços credenciados para realizá-la, à negativa de serviços públicos e à operacionalização, às vezes equivocada, de requisitos previstos na lei que a regulamentou 22 . No entanto, outras análises devem ser realizadas para avaliar a possível influência da redução da fecundidade e de mudanças nos padrões reprodutivos sobre a demanda por laqueadura na cidade de São Paulo. De fato, a fecundidade manteve-se em declínio na maioria dos distritos da cidade entre 2010 e 2014 e, em apenas sete dos 96 distritos, mantém-se acima do nível de reposição (2,1 filhos por mulher). Além disso, o adiamento do início da maternidade já se verifica em alguns deles, com mulheres tendo filhos após os 25 anos de idade 23 .

Observações da prática contraceptiva no mundo indicam tendência de declínio da esterilização feminina e masculina e incremento no uso de hormonais injetáveis e preservativo masculino nas últimas duas décadas. No entanto, em países que alcançaram taxas de prevalência da contracepção superiores a 60\%, métodos definitivos e os de longa duração (LARC sigla em inglês para long-acting reversible contraceptives) têm tido papel proeminente 24 . Na América Latina, por sua vez, há baixa frequência de uso de LARC. Estudo comparativo de 23 países da região com pesquisas nacionais realizadas entre 2008 e 2015 verificou que, em 17, menos de 10\% das mulheres em idade fértil, sexualmente ativas, usavam LARC, sendo o Brasil um deles 25. Apesar de o DIU ter sido introduzido no Sistema Único de Saúde (SUS) há mais de 30 anos e nos planos de saúde há 10 anos, seu uso é ainda muito restrito. No Município de São Paulo, ele não está disponível em todas as unidades de saúde do SUS 26 e nem sempre há profissionais aptos e interessados em ofertá-lo às mulheres 27 . Por outro lado, a preocupação com a proteção contra HIV e outras DSTs se mostrou concreta com a proporção expressiva de mulheres que usam pílula associada ao preservativo masculino (dupla proteção), principalmente entre as mais jovens.

O número de filhos tidos e a idade foram as principais variáveis associadas aos métodos em uso, indicando que a etapa da vida reprodutiva em que a mulher se encontra direciona sua opção contraceptiva dentro dos limites do que efetivamente está a seu dispor. Dessa forma, entre as mulheres sem filhos, predominam métodos reversíveis: pílula e preservativo masculino, e a idade modula a participação relativa de cada um deles.

Para as mulheres com 1 a 2 filhos, embora a pílula se mantenha como prática mais frequente, a vasectomia e a laqueadura tornam-se presentes no mix, principalmente entre as com 30 a 44 anos. Muitas devem ter completado sua intenção reprodutiva, uma vez que a fecundidade total estimada para o Município de São Paulo foi de 1,8 filho por mulher em 2014 23. Diferentemente, entre as que têm menos de 30 anos, aumenta a frequência de uso dos injetáveis. 
Conforme o esperado, quase $90 \%$ das usuárias de contracepção com 3 ou mais filhos tinham mais de 29 anos e haviam majoritariamente aderido aos métodos cirúrgicos. Só nessa situação, a escolaridade atuou como diferencial, mostrando participação comparativamente maior da vasectomia e menor da laqueadura entre mulheres com maior escolaridade, similar à situação encontrada em Campinas (São Paulo) em 2005 28,29 e em São Leopoldo (Rio Grande do Sul), em 201530.

\section{Conclusões}

A prevalência da anticoncepção entre mulheres sexualmente ativas é alta no Município de São Paulo, e as principais razões referidas para não estar praticando contracepção foram ausência ou inconstância de relações sexuais e o desejo de engravidar. De fato, os principais fatores associados ao não uso de contraceptivos entre mulheres que não queriam engravidar foram não ter parceiro e não ter tido relações sexuais nos 30 dias anteriores à entrevista. Seguiram-se a esses, o não uso de anticoncepção na primeira relação sexual, a ausência de consulta ginecológica no último ano, ter menos do que 3 filhos e a religião Pentecostal.

O mix contraceptivo em uso modificou-se quando comparado ao encontrado no Brasil em 2006. A prática, até então centrada em pílula e laqueadura no Brasil, está aqui concentrada em pílula e preservativo masculino, seja em uso isolado ou combinado. Os principais diferenciais dos tipos de métodos em uso foram o número de filhos tidos e a idade da mulher.

A pílula foi a prática mais frequente entre mulheres sem filhos e entre as que tinham até dois, seguida pelo uso do preservativo masculino, isolado ou a ela combinado. Nesses dois grupos de paridade, a idade modulou a participação relativa desses dois contraceptivos, mas a pílula se manteve como o método mais frequente em todos, exceto entre as mulheres sem filhos com 15 a 19 anos, em que foi superada pelo preservativo masculino.

Por outro lado, entre as mulheres com 3 ou mais filhos, a laqueadura foi a prática mais frequente, embora tenha sido superada pelo uso de hormonais injetáveis no grupo etário de 20 a 29 anos. Entre as mulheres com 30 a 44 anos, a maior escolaridade se associou a um mix no qual a laqueadura permaneceu mais frequente, porém com maior participação relativa do preservativo masculino e da vasectomia.

Em síntese, é possível dizer que o perfil de práticas contraceptivas observado indica o menor recurso à esterilização feminina, reflete a preocupação de uma parcela das mulheres com a dupla proteção, que o uso dos hormonais injetáveis se tornou mais frequente e que aumentou a participação masculina. No entanto, o DIU, método altamente eficaz e que pode se constituir na única alternativa de LARC não hormonal, segue sendo muito pouco utilizado, provavelmente por ser pouco ofertado.

$\mathrm{O}$ uso de hormonais apresentados na forma de adesivos, anéis vaginais e implantes são pouco acessíveis, uma vez que não estão disponíveis no SUS.

É preciso recolocar a anticoncepção na agenda de prioridades em saúde e direitos reprodutivos no âmbito da sociedade e dos formuladores de políticas públicas. É igualmente importante desenvolver novos estudos para verificar a possível influência de denominações neopentecostais sobre a decisão das mulheres em não praticar anticoncepção. Afinal, a autorregulação da reprodução, de forma efetiva e segura para sua saúde, é questão fundamental para o desenvolvimento de um projeto autônomo de vida da mulher. Na ausência dessa possibilidade, o mais provável é que a reprodução venha a determinar o curso de vida da mulher. 


\section{Colaboradores}

T. D. G. Lago coordenou o desenho e a implementação do estudo, processou e analisou os resultados e elaborou a primeira versão do artigo. S. Kalckmann participou de todas as etapas de desenho e implementação do estudo, do processamento e análise dos dados e da redação do artigo. M. C. G. P. Alves e M. M. L. Escuder foram responsáveis pelo desenho da amostra e ponderação do banco de dados, participaram da análise dos resultados e da redação do artigo. M. Koyama realizou as análises multivariadas e participou da redação do artigo. R. M. Barbosa participou do desenho e da implementação do estudo, da análise dos resultados e da redação do artigo.

\section{Informações adicionais}

ORCID: Tania Di Giacomo do Lago (0000-00029223-6672); Suzana Kalckmann (0000-0002-07111235); Maria Cecília Goi Porto Alves (0000-00034357-9473); Maria Mercedes Loureiro Escuder (0000-0002-3103-2617); Mitti Koyama (00000003-2518-8823); Regina Maria Barbosa (00000002-3390-2137).

\section{Agradecimentos}

Ao Departamento de Ciência e Tecnologia (DECIT)/Ministério da Saúde, Programa de Pesquisa para o SUS da Fundação de Amparo à Pesquisa do Estado de São Paulo (PPSUS/FAPESP; processo 2014/50115-1) e Organização Pan-Americana da Saúde (OPAS) pelo apoio financeiro.

\section{Referências}

1. Berquó ES, Oliveira MCAF, Camargo CPF, organizadores. A fecundidade em São Paulo: características demográficas, biológicas e socioeconômicas. São Paulo: Editora CEBRAP; 1977.

2. Nakamura MS, Morris L, Janowitz B, Anderson JE; Fonseca JB. Contraceptive use and fertility levels in Sao Paulo State, Brazil. Stud Fam Plann 1980; 11:236-46.

3. Costa AM. Participação social na conquista das políticas de saúde para mulheres no Brasil. Ciênc Saúde Colet 2009; 14:1073-83.

4. Lago TDG, Barbosa RM, Kalckmann S, Villela W. Acceptability of the diaphragm among lowincome women in São Paulo, Brazil. Int Fam Plan Perspect 1995; 21:114-8.

5. Kalckmann S, Lago TDG, Barbosa RM, Villela W, Goihman S. O diafragma como método contraceptivo: a experiência de usuárias de serviços públicos de saúde. Cad Saúde Pública 1997; 13:647-57.

6. Faúndes A, Cecatti JG. A operação cesárea no Brasil: incidência, tendências, causas, consequências e proposta de ação. Cad Saúde Pública 1991; 7:150-73.

7. Arruda JM, Rutemberg N, Morris L, Ferraz EA. Pesquisa nacional sobre saúde materno-infantil e planejamento familiar. PNSMIPF Brasil, 1986. Rio de Janeiro: Sociedade Civil Bem-Estar Familiar no Brasil/Instituto para Desenvolvimentod e Recursos; 1987.

8. Sociedade Civil Bem-Estar Familiar no Brasil. Pesquisa Nacional sobre Demografia e Saúde, 1996. Rio de Janeiro: Sociedade Civil Bem-Estar Familiar no Brasil; 1997.

9. Brasil. Lei no 9.263, de 12 de janeiro de 1996. Regula o $\$ 7$ o do art. 226 da Constituição Federal, que trata do planejamento familiar, estabelece penalidades e dá outras providências. Diário Oficial da União 1996; 15 jan.

10. Berquó E, Garcia S, Lago TDG. Pesquisa Nacional de Demografia e Saúde da Criança e da Mulher, PNDS 2006: relatório final. São Paulo: Centro Brasileiro de Análise e Planejamento; 2008.

11. Perpétuo IHO, Wong LLR. Desigualdade socioeconômica na utilização de métodos anticoncepcionais no Brasil: uma análise comparativa com base nas PNDS 1996 e 2006. In: Ministério da Saúde, organizador. Pesquisa Nacional de Demografia e Saúde da Criança e da Mulher PNDS 2006: dimensões do processo reprodutivo e da saúde da criança. Brasília: Ministério da Saúde/São Paulo: Centro Brasileiro de Análise e Planejamento; 2009. p. 88-104.

12. Coordenação de Epidemiologia e Informação, Secretaria Municipal da Saúde. Boletim CEInfo Saúde em Dados 2018; XVII(17). https:// www.prefeitura.sp.gov.br/cidade/secretarias/ upload/saude/arquivos/publicacoes/Boletim_ CEInfoDados_2018.pdf. 
13. Olsen JM, Lago TG, Kalckmann S, Alves M CGP, Escuder MML. Práticas contraceptivas de mulheres jovens: inquérito domiciliar no Município de São Paulo, Brasil. Cad Saúde Pública 2018; 34:e00019617.

14. Demographic and Health Surveys. DHS7 tabulation plan for key indicators report . https://dhsprogram.com/pubs/pdf/DHSM5/ Key_Indicators_Report_Tabulation_Plan_ 20Mar2015_DHSM5.pdf (acessado em 15/ Mar/2019).

15. Breiman LJH, Friedman RA, Stone CJ. Classification and regression trees. Belmont: Chapman \& Hall; 1984.

16. Sedgh G, Hussain R. Reasons for contraceptive nonuse among women having unmet need for contraception in developing countries. Stud Fam Plann 2014; 45:151-69.

17. Berquó ES, Barbosa RM, Lima LP; Grupo de Estudos em População, Sexualidade e AIDS. Trends in condom use: Brazil:1998 and 2005. Rev Saúde Pública 2008; 42 Suppl 1:S34-44.

18. Alves JED, Cavenaghi SM, Barros LFW. A transição religiosa brasileira e o processo de difusão das filiações evangélicas no Rio de Janeiro. Dossiê: religião e demografia. Horizonte - Revista de Estudos de Teologia e Ciências da Religião 2014; 12:1055-85.

19. Alves JED. A transição religiosa na cidade de São Paulo: 1991-2030. http://www.ihu.unisinos.br/78-noticias/574946-cidades-de-saopaulo-onde-os-evangelicos-ultrapassaram-oscatolicos (acessado em 15/Mar/2019).

20. Bastos LL, Brandão ER, Ventura M. Saúde sexual e reprodutiva, conservadorismo religioso e acesso a medicamentos: uma discussão sobre a estratégia global de advocacy do Consórcio Internacional sobre Contracepção de Emergência. Sex Salud Soc (Rio J.) 2017; 26:306-27.

21. Amorim FA, Bonifácio GMO. Tendências e diferenciais na prevalência dos métodos contraceptivos: uma análise a partir das DHS's realizadas no Brasil. In: Anais do XII Encontro Nacional de Estudos Populacionais 2010. http://www.abep.org.br/publicacoes/index. $\mathrm{php} /$ anais/article/view/2574/2504 (acessado em 15/Mar/2019)
22. Caetano AJ. Esterilização cirúrgica feminina no Brasil, 2000 a 2006: aderência à lei de planejamento familiar e demanda frustrada. Rev Bras Estud Popul 2014; 31:309-31.

23. Yazaki LM. Diferenciais Regionais de Fecundidade no Município de São Paulo. SP Demográfico 2016; 16(2). http://www.seade.gov. br/produtos/midia/2016/06/N.2_jun2016final-1.pdf.

24. Joshi R, Khadilkar S, Patel M. Global trends in use of long-acting reversible and permanent methods of contraception: seeking a balance. Int J Gynaecol Obstet 2015; 131 Suppl 1:S60-3.

25. Leon RGP, Ewerling F, Serruya SJ, Silveira MF, Sanhueza A, Moazzam A, et al. Contraceptive use in Latin America and the Caribbean with a focus on long- acting reversible contraceptives: prevalence and inequalities in 23 countries. Lancet Glob Health 2019; 7:227-35.

26. Borges ALV, Santos OA, Araújo KS, Gonçalves RFS, Santa Rosa PLF, Nascimento NC. Satisfação com o uso de métodos contraceptivos entre usuárias de unidades básicas de saúde da cidade de São Paulo. Rev Bras Saúde Matern Infant 2017; 17:757-64.

27. Figueiredo R, Castro JM, Kalckmann S. Planejamento familiar e reprodutivo na Atenção Básica do Município de São Paulo: direito constitucional respeitado? BIS Bol Inst Saúde 2014; 15:81-93.

28. Carvalho LEC, Osis MJD, Cecatti JC, Bento SF, Márcia B. Manfrinati MB. Esterilização cirúrgica voluntária na Região Metropolitana de Campinas, São Paulo, Brasil, antes e após sua regulamentação. Cad. Saúde Pública 2007; 23:2906-16.

29. Carvalho LEC, Cecatti JG, Osis MJD, Sousa H. Número ideal de filhos e arrependimento pós-laqueadura. Rev Assoc Med Bras 2006; 52:293-7.

30. Gonçalves TR, Leite HM, Bairros FS, Olinto MTA, Barcellos NT, Costa JSD. Desigualdades sociais no uso de contraceptivos em mulheres adultas no Sul do Brasil. Rev Saúde Pública 2019; 53:28. 
Abstract

Contraception is essential for women to be able to regulate their fertility, exercising a key dimension of reproductive rights. However, little is known about how women deal with this challenge in Brazil's largest city, São Paulo. To fill this gap, the population survey Ouvindo Mulheres: Contracepção no Município de São Paulo was conducted with a probabilistic sample of 4,000 women 15 to 44 years of age living in this city in 2015. This article presents the prevalence of contraceptive practice and analyzes factors associated with lack of contraception use and with types of contraceptives. Prevalence of contraception was estimated for women with at least one heterosexual relation in the 12 months prior to the interview and who were not pregnant. Logistic regression was used to verify factors associated with lack of contraception use, and the CHAID model was used to identify associations with the types of contraceptives used. Prevalence of contraception was 84.8\% (95\%CI: 83.2-86.3). The most prevalent contraceptives were the pill and condoms. Factors associated with lack of contraceptive use were religion (Pentecostal), number of children (fewer than 3), not having used contraceptives in the first sexual relation, not having a partner, and not having had sex in the previous month. Number of children and woman's age were the first two levels of discrimination of the types of contraceptives used. Prevalence of contraception was high, but maintaining a concentration in two methods: historically, female sterilization and the pill prevailed, nowadays, the pill and condoms do. New hormonal contraceptives should be incorporated by the Brazilian Unified National Health System (SUS), besides promoting the use of long-acting methods such as IUDs.

Contraception; Sexual and Reproductive Rights; Demographic Surveys

\section{Resumen}

La contracepción es fundamental para que las mujeres puedan regular su fecundidad, ejerciendo una de las dimensiones de sus derechos reproductivos. No obstante, desconocemos cómo enfrentan este desafio en la mayor ciudad de Brasil, São Paulo. Para resolver esta cuestión, se realizó la encuesta poblacional Ouvindo Mulheres: Contracepção no Município de São Paulo, mediante una muestra probabilistica de 4 mil mujeres de 15 a 44 años de edad, residentes en esa ciudad en 2015. En este artículo se presenta la prevalencia de la práctica contraceptiva, se analizan los factores asociados con el no uso de métodos anticonceptivos, así como los tipos de contraceptivos en uso. La prevalencia de la anticoncepción se estimó en mujeres con por lo menos una relación heterosexual, en los 12 meses anteriores a la entrevista, y que no estaban embarazadas. Se utilizó la regresión logística para verificar factores asociados al no uso de contracepción y el modelo CHAID para identificar asociaciones respecto a los tipos de contraceptivo en uso. La prevalencia de la anticoncepción fue 84,8\% (IC95\%: 83,2-86,3). Los contraceptivos más prevalentes fueron la píldora y el condón. Se asociaron al no uso de anticonceptivos: religión (Pentecostal), número de hijos (menos de 3), no haber usado contraceptivo en la primera relación sexual, no tener pareja y no haber tenido relaciones sexuales durante el mes anterior. El número de hijos y la edad de la mujer fueron los dos primeros niveles de discriminación de los tipos de contraceptivo utilizados. La prevalencia de la anticoncepción es alta, pero se mantiene la concentración en dos métodos: anteriormente, ligadura y píldora, ahora, píldora y condón. Es necesario incorporar nuevos contraceptivos hormonales en el Sistema Único de Salud (SUS), así como promover el uso de métodos de larga duración como el DIU.

Anticoncepción; Direchos Sexuales y Reproductivos; Encuestas de Población
Recebido em 31/Mai/2019

Versão final reapresentada em 05/Mar/2020 Aprovado em 09/Mar/2020 